San Jose State University

SJSU ScholarWorks

Doctoral Projects

Master's Theses and Graduate Research

Spring 5-1-2021

\title{
The effects of telehealth on patient satisfaction and information recall for breast cancer survivors during COVID-19
}

Stacey Teicher

California State University, Northern California Consortium Doctor of Nursing Practice

Follow this and additional works at: https://scholarworks.sjsu.edu/etd_doctoral

Part of the Other Nursing Commons

\section{Recommended Citation}

Teicher, Stacey, "The effects of telehealth on patient satisfaction and information recall for breast cancer survivors during COVID-19" (2021). Doctoral Projects. 136.

DOI: https://doi.org/10.31979/etd.ervj-96r3

https://scholarworks.sjsu.edu/etd_doctoral/136

This Doctoral Project is brought to you for free and open access by the Master's Theses and Graduate Research at SJSU ScholarWorks. It has been accepted for inclusion in Doctoral Projects by an authorized administrator of SJSU ScholarWorks. For more information, please contact scholarworks@sjsu.edu. 


\begin{abstract}
Project title: The effects of telehealth on patient satisfaction and information recall for breast cancer survivors during COVID-19
\end{abstract}

The coronavirus (COVID-19) pandemic has changed how cancer survivorship care is delivered. (Al-Shamsi, 2020). To protect vulnerable breast cancer patients, health care providers are moving towards telecommunication such as telephone, video, and secure messaging (electronic mail) versus in-person visits to connect with their patients (Segelov et al., 2020) during this pandemic. Breast cancer survivorship visits can be safely deferred from in-person visits to telehealth visits (Dietz et al., 2020). This doctoral project examines the impact of transitioning from in-person to telehealth (telephone or video) visits on patient satisfaction and recall of information for breast cancer survivors reviewing their Survivorship Care Plan (SCP) at an oncology clinic in Northern California. 
The effects of telehealth on patient satisfaction and information recall for breast cancer survivors during COVID-19

by

Stacey Teicher

A project submitted in partial fulfillment of the requirements for the degree of Doctor of Nursing

Practice

San Jose State University

Doctor of Nursing Practice

May 1, 2021 


\section{ACKNOWLEDGEMENTS}

"Knowing Is Not Enough; We Must Apply. Wishing Is Not Enough; We Must Do." - Johann Wolfgang Von Goethe

I thank my chair, Dr. Robin Whitney, for her endless time, expertise, and patience. I thank the entire staff at SJSU for their dedication and understanding, especially Dr. Ruth Rosenblum. My committee members, Dr. Raymond Liu and DNP Denise Prizznick for being so supportive and encouraging. I would like to acknowledge my fellow students, the first DNP cohort at SJSU, for our newfound friendships and amazing support - I am proud to know each and everyone of you and hope you all remain in my life well past this program. I would like to thank my KP colleague Elaine Jang, NP for supporting me with gathering surveys for my project - she helped make this all possible.

I could not have pursued this goal without the love and support of my girls Lily and Sophia. My teenager taught me how to navigate google docs, for which I am forever grateful. They saw me struggle and grow and persevere, and most importantly, never give up. My inspiration, Mary Lou Lewandowski, $\mathrm{PhD}$ at SJSU, was like a mother to me and she would be so proud to know I have accomplished this goal. Lastly, thank you to family and friends for their undying support and encouragement. 


\section{The effects of telehealth on patient satisfaction and information recall for breast cancer survivors during COVID-19}

\section{Background}

This doctoral project examines the impact of transitioning from in-person to telehealth (telephone or video) visits on patient satisfaction and recall of information for breast cancer survivors reviewing their Survivorship Care Plan (SCP) with a nurse practitioner. The coronavirus (COVID-19) pandemic has changed how cancer survivorship care is delivered (AlShamsi, 2020). To protect vulnerable breast cancer patients, health care providers are moving towards telecommunication such as telephone, video, and secure messaging (electronic mail) versus in-person visits to connect with their patients (Segelov et al., 2020) during this pandemic. A review of existing literature on the impact of COVID-19 on cancer survivors note the transition of survivorship visits to telehealth during the pandemic and the need for further research to define how telehealth has impacted patients physical, psychosocial and financial wellbeing (Dietz et al., 2020). Kaiser Permanente Northern California (KPNC) is an integrated health system serving $>4.5$ million patients across 21 hospitals and $>200$ outpatient facilities, with nearly a decade of experience in telehealth. Early experience during the pandemic at KPNC showed a rapid increase in telehealth in all aspects of oncology care, making this system ideal for research in telehealth and cancer survivorship (Neeman et al., 2020). Overall trends in telehealth encounters during January-March 2020 showed a $154 \%$ increase in telehealth visits when compared to 2019 (Koonin et al., 2020). Little research has been done to know if breast cancer survivors are satisfied with telehealth for survivorship visits and their level of comfort doing self-breast exams (SBE). Awareness and comfort of performing regular SBE's can potentially detect early-stage breast cancer and increase chance of survival (Kumarasamy et al., 
2017). A study by Huguley \& Brown (1981) found $76 \%$ of women under the age of 50 performed SBE's, and $68 \%$ between the ages of $50-69$, yet only $50 \%$ of women over the age of 70 performed SBE's. The purpose of this project is to evaluate the impact of telehealth survivorship visits on patient satisfaction and information recall during COVID-19 pandemic. The findings could impact how survivorship care is delivered long after the pandemic is over.

\section{Literature Review}

\section{Needs of Breast Cancer Survivors}

Breast cancer is the leading cause of cancer in women (Iacoviello et al., 2020). Female breast cancer has a survival rate of nearly $90 \%$ at the five- year mark following cancer treatment (Runowicz, 2016). Although a person is considered a cancer survivor from the time of diagnosis until the end of life (Doyle, 2008), survivors have unique physical, psychological, and social needs after active care has ended. The Institute of Medicine acknowledges the importance of surveillance, health promotion, and management of physical, psychological, spiritual, social, and practical long-term and late effects that many survivors of cancer face after completing active treatment (Runowicz, 2016).

To address these needs, the Institute of Medicine (IOM) and National Research Council of the National Academies (2005) recommend oncology providers create a survivorship care plan (SCP) for the cancer patient, and their primary care provider (PCP), after completion of treatment. The purpose of a SCP is to ensure proper surveillance care for cancer survivors (Jacobs et al., 2017). The American College of Surgeons (ACOS) Commission on Cancer (CoC) has adopted SCPs as a standard of care for breast cancer survivors (ACOS, 2018). The SCP should include a list of all treating providers, a summary of treatment received (surgery, 
radiation, chemotherapy, and endocrine therapy), recommended follow-up schedule (clinic visits and surveillance tests), healthy lifestyle recommendations (exercise, nutrition, supplements), and education on detecting signs of possible recurrence (National Research Council, 2005). Information on endocrine therapy and medical information regarding long term side effects from their treatments should be included, if applicable. At the time the patient receives the SCP, the provider will also offer additional resources as needed, such as referrals for social work, dietician, physical therapy (lymphedema clinic), acupuncture, and support groups. A comprehensive SCP provides the patient and PCP with a written record of their past care and treatment(s) received, possible long-term side-effects from the treatment and how to manage them, and a surveillance schedule.

\section{Impact of COVID-19 on Survivorship Care.}

Traditionally, post treatment follow-up to review the SCP with a breast cancer survivor patient has been done in person, but with the COVID-19 crisis, these in-person visits have been moved to telehealth visits. As of March 13, 2021, there are 121 million cases of COVID-19 worldwide, with 2.68 million deaths (World Health Organization, 2021). Telehealth visits protect healthcare workers and patients from being exposed to infection (Leite et al., 2020) which could help reduce the spread of COVID-19 and create less stress on the healthcare system. With the disease continuing to spread in the United States, COVID-19 could have long term consequences and change the way we deliver oncology survivorship care. Due to COVID-19, in-person visits have been prioritized to only life threatening or symptomatic conditions that require urgent treatment (Dietz, 2020).

\section{The Role of Telehealth}


Telehealth provides an opportunity for nurse practitioners to continue their survivorship clinics virtually by providing services, education, and managing symptoms while avoiding direct physical contact (Larson et al., 2019), decreasing risk for themselves and their patients. Telehealth provides patients with the convenience of staying home. Although telehealth provides many benefits, it raises challenges for healthcare practices that traditionally are done during in-person visits (Leite et al., 2020) and telehealth has been implemented in a fragmented way for many healthcare centers due to the urgency during this pandemic.

While previous work suggests that telehealth is generally easy to use, convenient, cost-effective, and associated with positive patient outcomes (Kruse et al., 2017), there are mixed findings regarding the effect of telehealth on quality of care for cancer survivors, specifically. A recent systematic review and meta-analysis study concluded telehealth has a statistically significant impact on quality-of-life scores compared to usual care among cancer patients (Larson et al., 2019). However, a study done in 2011 surveyed 218 breast cancer survivors and the results found that patients rated virtual visits as less favorable than in-person visits (Mayer et al., 2011). It is possible that telehealth visits work well for some cancer survivors, but not others. An evaluation of telehealth survivorship care plan visits among breast cancer patients found that nearly half preferred telehealth to in-person visits, citing reduced costs associated with co-pays, transportation, and time off work as key factors for preferring telehealth (Washington et al., 2017). COVID-19 has added another incentive to use telehealth for safety reasons. A survey done at the Houston Methodist Cancer Center collected 1477 responses regarding telehealth satisfaction, and $92.6 \%$ patients reported satisfaction (Darcourt et al., 2021), which demonstrates the potential of telehealth to further improve interactions between providers and their cancer patients without lessening the quality of care (Darcourt et al., 2021). 
To assure high quality care, survivorship clinics should evaluate the impact of telehealth on their patients with respect to key outcomes, including patient satisfaction and information recall. Patient satisfaction is important for telehealth to be "a viable mode of service delivery" (Orlando et al., 2019). Satisfaction with health care is associated with improved treatment compliance and patient engagement (Orlando et al., 2019). Positive health outcomes, communication, satisfaction with technology, education and information provided, reduced need to travel, and lower costs (Hilgart et al., 2012; Kruse et al., 2017) all contribute to patient satisfaction. However, face-to-face interactions, written handouts, and the ability to ask questions and repeat what was told to them, may contribute to better patient information recall (Kruse et al., 2017). If patients cannot recall information given to them, they are less likely to adhere to health recommendations (Watson \& Mckinstry, 2009). Therefore, evaluating the impact of the shift to telehealth on information recall during these visits is critical.

\section{Study Purpose}

The purpose of this DNP project is to evaluate the impact of implementing telehealth visits in a breast cancer survivorship clinic. The aims are to 1) evaluate patient satisfaction and 2) assess patients' ability to recall information received after completing a telehealth survivorship visit.

\section{Theoretical Framework}

Quality of medical care is difficult to measure, yet it is important to evaluate. The Donabedian theory uses seven elements to measure quality of medical care: efficacy, 
effectiveness, efficiency, equity, optimality, acceptability, and legitimacy (Ameh $e t$ al.,2017). Donabedian's definition of quality of care is based on structure, process, and outcome. "Good structure should promote good process and good process should in turn promote good outcome" (Ameh et al., 2017). According to Donabedian's framework, structure is defined as accessibility, availability, and quality of resources. In terms of telehealth, structure includes factors like patients' access to telehealth (do they have internet or a smartphone), and providers' availability to provide care through telehealth. Process is the delivery of health care services by providers. For example, provider breast exams must be changed to self-breast exams with the use of telehealth versus in-person. Outcomes are final results of health care services and how it impacts the individual and the organization. In the context of telehealth, this could include patient and provider satisfaction and comfort with technology, and patient recall of important information delivered during the visit. Donabedian's framework posits that structure influences process, which influences outcomes. COVID-19 has changed the structure of survivorship care by shifting care delivery from in-person to virtual (telehealth), therefore it is important to examine how these changes ultimately impact quality of care.

\section{Study Design}

\section{Methods}

This quality improvement evaluation was a descriptive study to evaluate the impact of telehealth survivorship visits on patient satisfaction and information recall, with telehealth visits as an existing standard of care. The study was submitted and approved as a quality improvement project by Kaiser Permanente's Research Determination Committee. This project was conducted as a quality improvement project, and therefore was not supervised by the 
institutional review board. Three Northern California nurse practitioner run survivorship clinics participated in the study. The Diablo Service Area includes clinics in Antioch, Dublin, and Walnut Creek in Northern California.

\section{Sample}

Our sample consisted of female breast cancer survivors who had a telephone or video appointment in one of the three NP-run survivorship clinics in the DSA from August 15, 2020 until December 15, 2020. The study included all breast cancer survivors' stages 0 - III who have completed treatment for their breast cancer and participated in an initial 40-minute survivorship care appointment to review their SCP with the nurse practitioner.

\section{Procedures}

The telehealth cancer survivorship visit is a one-time, 40-minute appointment via telephone or video to review the patient's SCP. The SCP template was created by Kaiser Permanente in accordance with ACOS requirements (ACOS, 2015) which outlines the specific information that an SCP should include. The template pulls information from the patient's electronic chart such as the patient's care team and treatment summary. Included is the follow up care plan, healthy life-style recommendations, possible effects from their cancer treatments, and a list of resources offered to them. The survivorship NP prepares the SCP and sends it to the patient via Kaiser Permanente's secure message system prior to their appointment.

\section{Data Collection}


An electronic patient survey (see appendix A) was developed which contained 20 questions related to patient satisfaction and education recall. All participating members who have agreed to do the survey and have kp.org received a survey via secure messaging through the health connect portal, and all inactive kp.org members received the same survey via telephone. Patients who prefer a language other than English were provided an interpreter to translate survey questions to their preferred language. A member of the project team (other than the NP who performed the visit) called the patient, introduced themselves, and asked the survey questions over the phone, following the telephone script (see Appendix C). Survey responses were entered directly into the Microsoft Forms survey.

Microsoft Forms was used to build the survey instrument, and this platform was used to collect responses on a secure and encrypted website. The study database with the collected responses was kept in a secure, password protected database, and all data were deidentified. Data were aggregated, and individual patient information was only accessed as part of the usual survivorship care for the patient. Data used for quality improvement efforts were reported out only in aggregate format.

\section{Measures}

The survey consisted of a total of 20 questions related to patient satisfaction, telehealth preferences, information recall and demographics. The full questionnaire is available in Appendix A. Patient satisfaction was measured with five questions adapted from the Visit Satisfaction Instrument (VSQ-9) using a 5-point scale (poor, fair, good, very good, and excellent) to measure patients' satisfaction with their oncology, with higher scores indicating higher satisfaction (RAND Health Care, 2018). 
Seven questions asked about telehealth preferences, including whether patients prefer telephone or video visits and why, if they like receiving information via email through kp.org (Kaiser Permanente secure email messaging), and if they are satisfied with telehealth (video or telephone). These tools were created for the purpose of this QI project and have no established reliability or validity. Six questions were related to information recall from the survivorship visit and covered each category reviewed by the nurse practitioner. Categories included cancer stage and type, follow up plan, healthy life-style recommendations, available resources, review of past treatments, and side effect management, if applicable. Six questions asked participants to rank each category discussed during their survivorship visit from most to least useful information. In transitioning to telehealth survivorship visits, patients must complete self-breast exams rather than provider breast exams. To assess comfort with this transition, we asked about patient comfort conducting self-breast exams, where patients answered yes or no. Patient demographic information collected included age $(<40 ; 41-55 ; 56-65 ; 66-75$; and $>75)$. Kaiser does not recommend mammograms after the age of 75 but is available to the patient if requested. Preferred written language was also asked.

\section{Data Analysis}

All analyses were conducted using Intellectus Statistics. (2019) and Microsoft Excel. Descriptive statistics (e.g., frequencies, proportions) were tabulated for all variables. A Spearman correlation analysis was conducted using four questions to examine which aspects of telehealth were associated with higher satisfaction: time spent with the NP, personal manner, convenience, and technical skills. Cohen's standard was used to evaluate the strength of the relationships, where coefficients between .10 and .29 represent a small effect size, coefficients 
between .30 and .49 represent a moderate effect size, and coefficients above .50 indicate a large effect size (Cohen, 1988). The result of the correlations was examined using Holm corrections to adjust for multiple comparisons based on an alpha value of 0.05 .

Summary statistics were calculated for information recall on cancer stage and type, resources, follow up plan, treatment review, side effects, life- style, and self-breast exams. A majority of patients were able to recall information that was discussed at their visits. Across the information recall items, the percentage of patients who could not remember discussing an item ranged from 6-14\%, with resources and the importance of breast self -exams being the most commonly forgotten. A majority of patients felt that the right level of information was provided for most items, ranging from $82 \%$ for discussion of long-term side effects to $92 \%$ for discussion about follow-up plans.

Chi-square Tests were used to examine whether comfort with self-breast exams differed by age group (>65 versus 65 and younger). A Spearman correlation analysis was conducted between patient age and overall satisfaction with the visit. There were no significant correlations between any pairs of variables.

\section{Results}

Data collection took place between August and December 2020 at 3 three clinics within a large health care system in Northern California. 93 surveys were sent electronically through secure kp.org secure mail and 7 surveys were conducted via telephone. Of the 100 surveys sent, 62 were completed. One patient declined to participate stating "I feel I am not good with surveys". Of the patients who requested to complete the survey via telephone, 6 were inactive on kp.org secure email, and 2 were Spanish speaking and required an interpreter. 


\section{Participant Characteristics}

Demographic data collected included preferred language and age. All participants were women. 97 participants were English speaking, 2 were Spanish speaking, and one spoke Tagolog. 5\% were less than age 40; $23 \%$ were between the ages of $41-55 ; 32 \%$ were between the ages of $56-65 ; 31 \%$ were between the ages of $66-75$; and $10 \%$ were greater than 75 years of age. $73 \%$ of the participants completed the survey within 4 weeks of their SCP visit, $18 \%$ within the last three months, and $8 \%$ greater than three months ago (Table 1).

Table 1. When was the survey done post initial survivorship visit.

\begin{tabular}{|c|c|c|}
\hline Time from SCP visit & number & percentage \\
\hline Within 4 weeks & 45 & $73 \%$ \\
\hline Within 3 months & 12 & $18 \%$ \\
\hline Greater than 3 months & 5 & $8 \%$ \\
\hline
\end{tabular}

\section{Patient Satisfaction}

Patients rated their level of satisfaction as excellent and very good with a mean of $86 \%$ for all categories. Overall satisfaction with their telehealth SCP visit was rated excellent by $85 \%$ and very good by $11 \%$ of participants. Convenience of the telehealth appointment rated lowest at $74 \%$ saying it was excellent, and $6 \%$ stating it was either fair or poor.

A significant positive correlation was observed between each visit characteristic analyzed and overall satisfaction, including time spent with the NP $\left(r_{\mathrm{s}}=0.78, p<.001,95 \%\right.$ CI $[0.65$, 0.87]), personal manner $\left(r_{\mathrm{s}}=0.66, p<.001,95 \%\right.$ CI $\left.[0.49,0.79]\right)$, convenience $\left(r_{\mathrm{s}}=0.78, p<\right.$ $.001,95 \%$ CI $[0.65,0.87])$, and technical skills $\left(r_{\mathrm{s}}=0.60, p<.001,95 \%\right.$ CI $\left.[0.40,0.74]\right)$. All 
effect sizes had a correlation coefficient between 0.60 and 0.78 , indicating a large effect size and suggesting that as each of these factors increase, satisfaction tends to increase.

\section{Table 2}

Spearman Correlation Results Among Overall satisfaction, Time spent with the NP, Personal manner, Convenience, and Technical skills

Zombination

$r_{\mathrm{S}}$

$95 \% \mathrm{CI}$

$p$

)verall satisfaction-Time spent with the NP

0.78

$[0.65,0.87]$

$<.001$

)verall satisfaction-Personal manner

0.66

$[0.49,0.79]$

$<.001$

)verall satisfaction-Convenience

0.78

$[0.65,0.87]$

$<.001$

Jverall satisfaction-Technical skills

0.60

$[0.40,0.74]$

$<.001$

\section{Information recall}

Overall most patients were able to recall information discussed at their visits. Across the information recall items, the percentage of patients who could not remember discussing an item ranged from 6-14\%, with resources and the importance of breast exams being the most commonly forgotten. Most patients felt that the right level of information was provided for most items, ranging from $82 \%$ for discussion of long-term side effects to $92 \%$ for discussion about follow-up plans (see table 3).

Table 3. Level of information.

\begin{tabular}{|c|r|r|}
\hline Variable & $n$ & $\%$ \\
\hline Healthy life- style recommendations & & \\
\hline Discussed, just the right level of information & 42 & 85.71 \\
\hline
\end{tabular}




\begin{tabular}{|c|c|c|}
\hline I do not remember & 3 & 6.12 \\
\hline Discussed, but not enough & 3 & 6.12 \\
\hline Discussed, too much information & 1 & 2.04 \\
\hline \multicolumn{3}{|l|}{ Long term side effects } \\
\hline Discussed, just the right level of information & 40 & 81.63 \\
\hline I do not remember & 3 & 6.12 \\
\hline Discussed, but not enough & 6 & 12.24 \\
\hline \multicolumn{3}{|l|}{ Cancer stage and type } \\
\hline Discussed, just the right level of information & 42 & 84.00 \\
\hline Discussed, but not enough & 4 & 8.00 \\
\hline I do not remember & 4 & 8.00 \\
\hline \multicolumn{3}{|l|}{ resources } \\
\hline Discussed, just the right level of information & 40 & 83.33 \\
\hline I do not remember & 7 & 14.58 \\
\hline Discussed, but not enough & 1 & 2.08 \\
\hline \multicolumn{3}{|l|}{ Importance of self-breast exams } \\
\hline Discussed, just the right level of information & 41 & 83.67 \\
\hline I do not remember & 7 & 14.29 \\
\hline Discussed, but not enough & 1 & 2.04 \\
\hline Follow-up plan & & \\
\hline Discussed, just the right level of information & 45 & 91.84 \\
\hline
\end{tabular}




\begin{tabular}{|c|r|r|}
\hline I do not remember & 2 & 4.08 \\
\hline Discussed, but not enough & 2 & 4.08 \\
\hline
\end{tabular}

\section{Self-breast exams}

Overall, $79 \%$ of patients answered that they were comfortable with doing self-breast exams (SBE), while $21 \%$ stated they were not comfortable. Among patients age 66 and above, $75 \%$ reported feeling comfortable doing SBE and $80 \%$ of patients age 65 and under reported being comfortable doing SBE. However, these differences were not statistically significant $\chi^{2}(1)$ $=0.32, p=.571($ Table 4$)$.

Table 4. Comfort of doing self-breast exams. $(n=61)$.

Observed and Expected Frequencies

\begin{tabular}{|c|r|r|r|r|r|}
\hline & \multicolumn{3}{|c|}{ Are you comfortable doing self-breast exams } & & \\
\hline age & Yes & No & $\chi^{2}$ & $d f$ & $p$ \\
\hline 65 and under & $30[29.11]$ & $7[7.89]$ & 0.32 & 1 & .571 \\
\hline 66 and older & $18[18.89]$ & $6[5.11]$ & & & \\
\hline
\end{tabular}

\section{Information ranking from most to least useful}

Survey question number 5 asked the participants to rank the information discussed during their survivorship visit from most to least useful. Most participants listed follow-up plan and cancer stage and type as their first choice, and healthy life-style recommendations and review of treatments and long-term side effects as their last choice (Figure 1).

Figure 1. Breast Cancer Survivor Ranking of Survivorship Visit Information from Most to Least Useful $(n=57)$. 


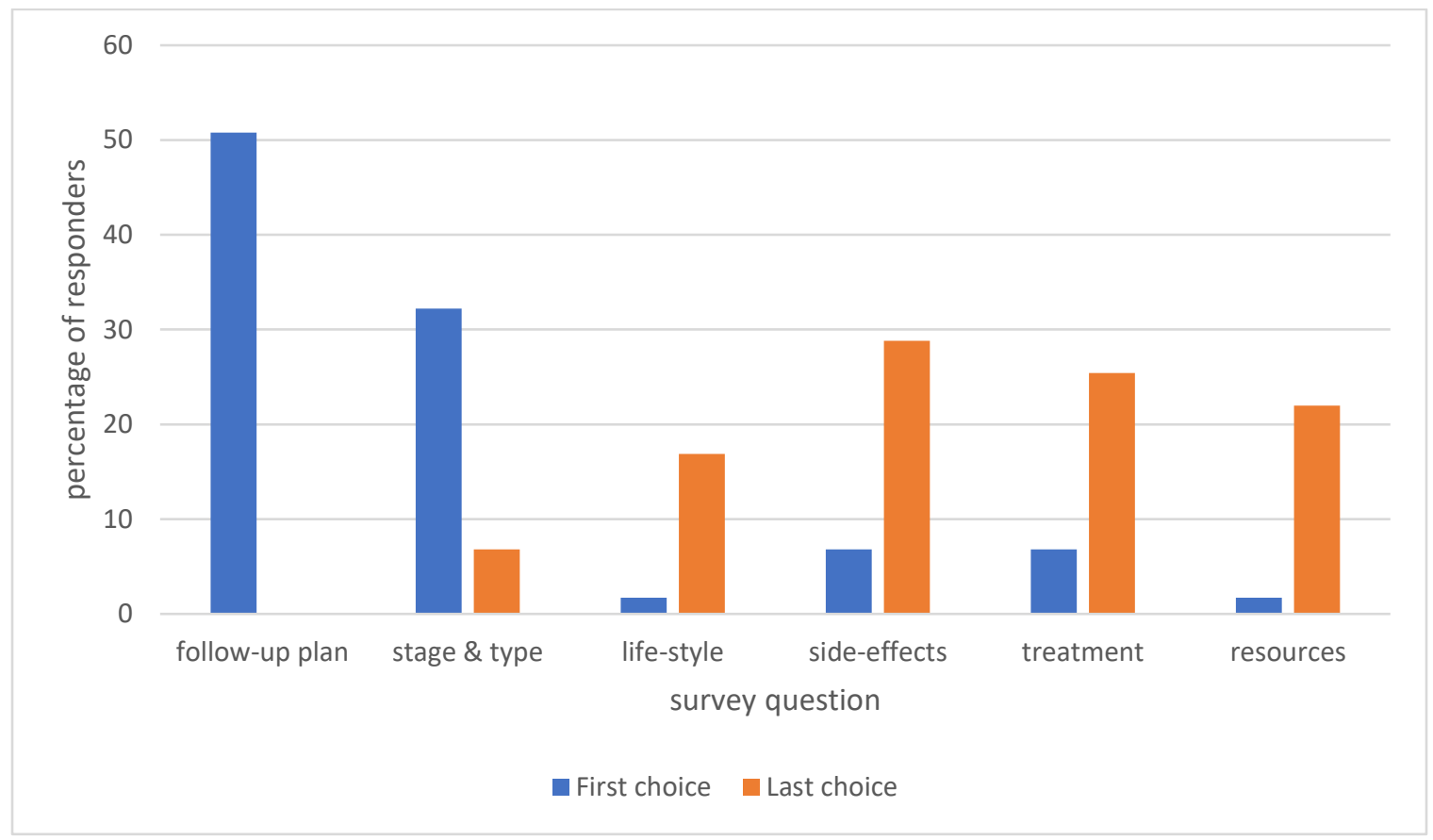

\section{Telehealth Satisfaction}

A large majority of patients reported that they were comfortable using telehealth $(80 \%)$ and liked getting email via the EHR (83\%). Only $32 \%$ of patients agreed that receiving a SCP in the mail would be acceptable in lieu of a video or telephone appointment (Figure 2). 
Figure 2. Telehealth Satisfaction Among Breast Cancer Survivors ( $(\mathbf{n}=59)$.

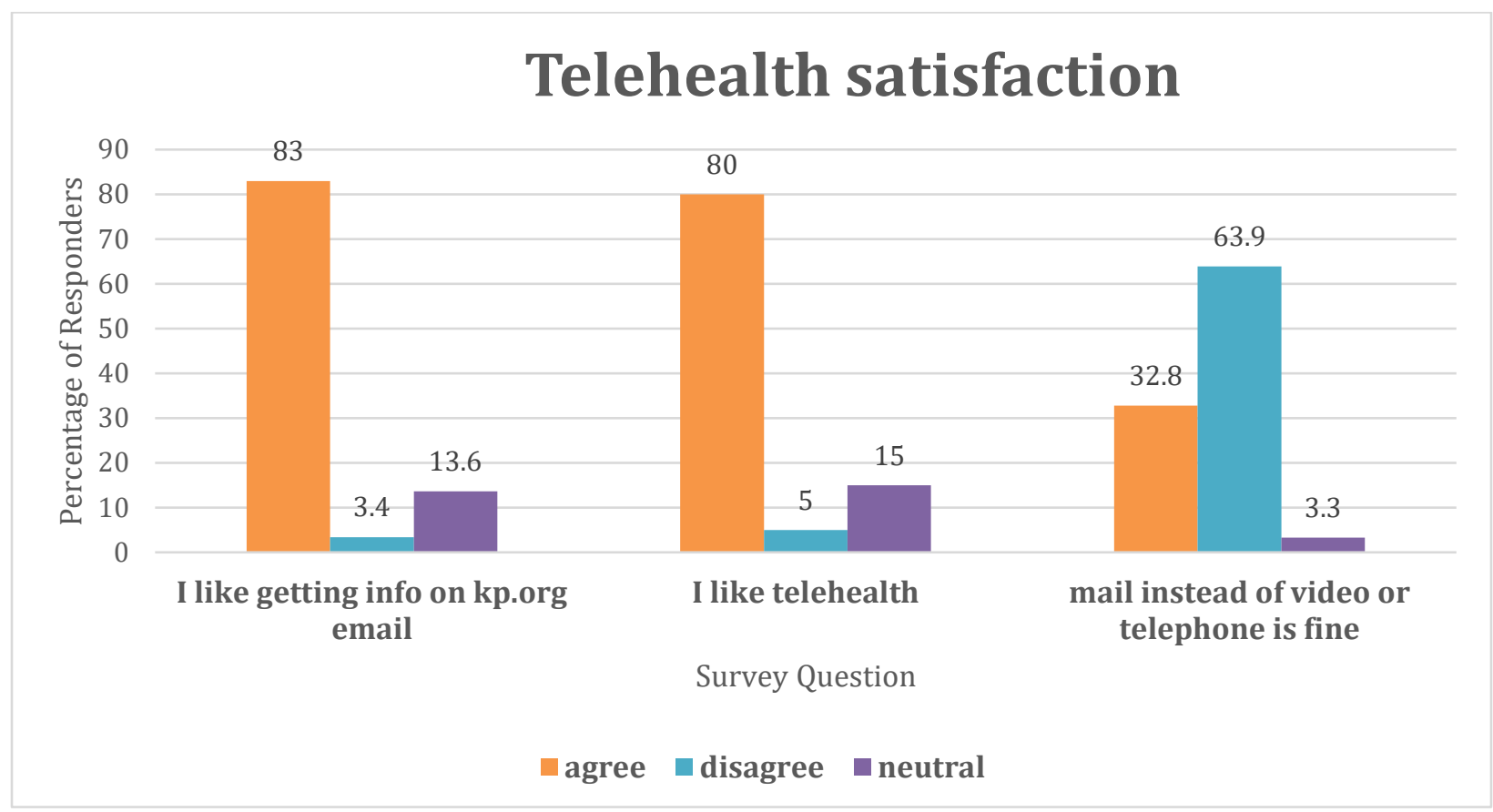

\section{Preferred form of Telehealth}

Fifty percent of respondents said they preferred telephone visits, while 50\% said they do not. $69 \%$ of respondents said they preferred video visits, and $31 \%$ did not prefer video visits. Appendix F lists the reported reasons for preferring telephone or video visits.

The most commonly reported reasons for preferring telephone visits were related to convenience, preferring to in-person during COVID-19, lack of computer access, and feeling that telephone is less of a hassle than video.

The most commonly reported reasons for preferring video appointments were related to feeling more personal because you can see the person you are talking to, feeling that video is easier than going in person, perceiving that communication is equal to in-person. 
A spearman correlation analysis was conducted between what is your age and overall satisfaction of the telehealth visit. The result of the correlation was examined based on an alpha value of 0.05 . There were no significant correlations between any pairs of variables. Table 5 presents the results of the correlation.

Table 5: Spearman Correlation Results Between What is your age and Overall satisfaction $(\mathbf{n}=\mathbf{5 9})$.

\begin{tabular}{|c|r|r|r|}
\hline Combination & $r_{s}$ & $95 \% \mathrm{Cl}$ & $p$ \\
\hline What is your age-Overall satisfaction & 0.10 & {$[-0.16,0.34]$} & .466 \\
\hline
\end{tabular}

\section{Discussion and Conclusion}

\section{Discussion}

In this quality improvement project evaluating satisfaction with telehealth survivorship visits, we identified several factors associated with higher satisfaction, including perceived convenience, time spent with the NP, technical skills of the NP, and personal manner of the NP. Overall satisfaction with their SCP visits via telehealth was high, with patients overwhelmingly rating the satisfaction as excellent. Our findings that patients are highly satisfied with telehealth survivorship care is similar to previous work. A pilot study comparing patient satisfaction and 
recall of diet and exercise recommendations from SCP's (Sprague \& Holschuh, 2019) between telehealth and in-person visits found that both satisfaction and information recall were similar in both formats. Darcourt et al., (2020); found that among oncology patients who were offered video visits during the COVID-19 pandemic, older patients were significantly less likely to participate. In contrast, we found no association between age and satisfaction with visits in our study., which challenges the idea that older populations are more resistant to telehealth. Reasons cited for preferring telehealth over face-to-face visits for older adults in our study included convenience, no travel time, and no co-pays.

A majority of patients in our study appeared to retain the information given during their SCP meeting and felt the right amount of information was provided. Sprague \& Holschuh (2019) found similar results showing no statistically significant differences in patient satisfaction or recall between cancer survivors receiving their survivorship care plan by telephone versus a clinic appointment.

Just over half of survivors in our study ranked the follow-up plan as the most useful information discussed during their survivorship visit. Patients are anxious to know what comes next once they are done with active treatment and when they enter the surveillance period, which is discussed and reviewed during the SCP visit. The second highest ranked was cancer stage and type, which $32 \%$ of patients identified as the most useful information discussed. This finding reinforces the need to review important information with patients on more than one occasion. A great deal of time is spent with cancer patients explaining their diagnosis and treatment options; and it may take several discussions for a patient to fully understand their diagnosis (Klevos et al., 2017). Patients initially learn about their cancer stage and type by their oncologist at the initial consultation, but patient anxiety and fears may prevent the patient from registering the 
information given to them. Continued education and reassurance can help to ease anxiety (Klevo set al., 2017) therefore it is not surprising that patients still want to hear about their cancer stage and type at the end of their treatments.

Interestingly, the lowest ranked discussion item was healthy life-style recommendations with only $1.7 \%$ of participants ranking it as most important. Healthy life-style actions such as adequate sleep, exercise, and good nutrition (Klevos et al., 2017) are vital to health promotion and to prevention of recurrence and secondary cancers. For example, research has shown that breast cancer patients who gain weight during or after their treatment are at higher risk of breast cancer related death (Hamer \& Warner, 2017). Diets high in saturated fats, processed meats, red meat, and processed grains may also be associated with higher breast cancer mortality (Hamer \& Warner, 2017) and should be avoided. Cancer survivors are motivated to seek information about diet and physical activity to improve their response to treatment and survival (Brown et al., 2003), but there are few studies that examine diet and exercise behaviors in cancer survivors after the completion of their cancer treatments. DeNysschen et al., (2013) conducted a secondary analysis on breast cancer patients who participated in a randomized control trial of healthy life-style behaviors based on the 2012 American Cancer Society (ACS) guidelines for healthy lifestyle behaviors. They found that during their cancer treatments and directly afterwards, the participants were highly motivated, but six months later, most participants reverted back to their previous behaviors (DeNysschen et al.,2013). Taken together, previous work showing that lifestyle modifications are key to maintaining good health in cancer survivors and our finding that most patients in our survey do not view information about lifestyle modification as very important suggest that further research is important in understanding how specific breast cancer populations prioritize different elements of their survivorship care. 
We found that a majority of patients are comfortable doing self-breast exams, which is an important change from the provider-conducted breast exams conducted during in-person visits. It is important to identify which patients are not comfortable doing breast exams and provide education for them on how to do a self-breast exam. Patients' who are comfortable doing breast exams may be less anxious to be seen for an exam during the pandemic.

More than half of the participants strongly agreed that they liked getting information from their providers through secure email and were comfortable doing survivorship visits through telehealth (31\% preferred telephone visits, and 69\% preferred video visits). Only $8.2 \%$ strongly agreed to email visits instead of telephone or video. Based on the responses to why they prefer telephone or video visits, they overwhelmingly felt they preferred having a dialog with their provider, and felt video was more personable. Trust in their provider was created when they could see them.

\section{Limitations}

Our study had several important limitations. As this was a quality improvement project, the main objective was to evaluate the results of telehealth implementation at our institution rather than to produce generalizable results. Although $62 \%$ of the surveys sent were completed, the overall sample size was small. It is possible that those who chose not to respond to the survey were less satisfied with telehealth than those who did, which would have caused us to overestimate patients' positive experiences with telehealth visits. Two weeks into the start of data collection, the NP running the Walnut Creek survivorship clinic retired, therefore only five surveys were completed from the Walnut Creek clinic. Survey questions regarding patient recall from their survivorship visit were designed to reflect the content of our institution's SCP template, with no reliability or validity testing done. $97 \%$ of our patients reported English was 
their preferred language, which may limit the applicability of our findings to more diverse clinics even within our own health systems. Cancer stage and exact age was not captured - these variables could affect satisfaction and recall.

\section{Implications for Research}

Future studies are needed on patient satisfaction and information recall of nurse practitioner led telehealth visits, focusing on cancer survivorship. The COVID-19 pandemic has steeply shifted health care delivery from face-to-face visits to telehealth. Telehealth can provide safe and appropriate care to a cancer patient while minimizing exposure to COVID-19 (Paterson, et al., 2020). Once oncology patients have started active treatment, many of the traditional inperson visits can be moved to telehealth such as supportive care, symptom management, and triage support (Neeman et al.,2020). There are multiple published articles on the effectiveness of telehealth for cancer care, but few on patients' satisfaction with telehealth care. Further research on telehealth satisfaction should be done for the feasibility of telehealth to continue post pandemic.

More longitudinal studies are needed to examine how cancer survivorship can contribute to monitoring and encouraging healthy life-style behaviors. Future research should focus on healthy life-style behaviors. For example, conducting qualitative interviews with cancer survivors to identify reasons why cancer survivors do not value healthy life-style recommendations, which may help to identify promising new approaches to encourage cancer survivors to make healthy lifestyle choices.

More in-depth studies comparing patient satisfaction with telephone versus video appointments would be beneficial to changing the way we deliver SCP to our patients after completing treatment for breast cancer. Telehealth can potentially extend well beyond SCP 
visits and be applied to all on and off treatment cancer patients. As the availability of telehealth services expands, studies should be performed to examine reluctance to use telehealth by both the providers and the patients. Barriers to the use of telehealth such as lack of proper equipment, mistrust, and lack of knowledge should also be studied.

\section{Implications for Nursing}

Telehealth allows nurses to provide education and survivorship care to cancer survivors during the COVID-19 pandemic safely and conveniently. Patients have shown they are satisfied with their SCP telehealth visits, which could potentially continue well after the pandemic is over. With the emergence of the U.S. Coronavirus Aid, Relief, and Economic Security (CARES) Act in March 2020, Medicaid and Medicare now includes Telehealth as a covered benefit, and waives all co-pays (Koonin et al., 2020). Evidence has shown that telehealth in cancer care has reduced travel burden, provides timely interventions to discuss side effects, initiate interventions, provide education and support while keeping the patient safely at home (Paterson et al., 2020). Telehealth may provide a long-lasting solution for cancer patients and may be adopted long-term for cancer survivorship. Nurses play a pivotal role in cancer care, caring for both the emotional and physical needs of the cancer survivor.

Nurse Practitioners are in a strong position to promote healthy life-style behaviors through the survivorship program. Breast cancer patients are followed regularly post-treatment therefore telehealth survivorship is an excellent platform to promote healthy behaviors well past their cancer treatments. Results from this survey indicate patients do not value information on healthy-lifestyle recommendations despite its importance. Nurse practitioners can incorporate healthy-lifestyle programs into the SCP and be on the forefront to encourage compliance longterm. 


\section{Conclusion}

This project examined the effects of telehealth on patient satisfaction and information recall for breast cancer survivors during COVID-19. The study results concluded there was a high level of patient satisfaction with their SCP visit via telehealth with their nurse practitioner, along with appropriate information recall. Telehealth may provide a long-lasting solution for cancer patients and may be adopted long-term for cancer survivorship. High comfort rate for patients doing their own breast exams may lead to more emphasis on self-breast exams rather than routine provider breast exams. More research on nurse practitioner-run survivorship programs via telehealth should be performed to support the role of the nurse practitioner in cancer survivorship care. 


\section{References}

Breast Cancer Survivorship Care. (2018). Retrieved from https://www.facs.org/qualityprograms/napbc/standards

From cancer patient to cancer survivor: Lost in transition. (2006). Washington DC: National Academies Press.

Al-Shamsi, H. O., Alhazzani, W., Alhuraiji, A., Coomes, E. A., Chemaly, R. F., Almuhanna, M., ... \& Meyers, B. M. (2020). A practical approach to the management of cancer patients during the novel coronavirus disease 2019 (COVID-19) pandemic: an international collaborative group. The oncologist, 25(6), e936.

Alfano, C. M., Jefford, M., Maher, J., Birken, S. A., \& Mayer, D. K. (2019). Building personalized cancer follow-up care pathways in the United States: lessons learned from implementation in England, Northern Ireland, and Australia. American Society of Clinical Oncology Educational Book, 39, 625-639.

American College of Surgeons Commission on Cancer. (2018). Cancer Program Standards: Ensuring Patient-Centered Care Manual (2016 Edition). Standard 3.2-Psychosocial Distress Screening.

Ameh, S., Klipstein-Grobusch, K., D’ambruoso, L., Kahn, K., Tollman, S. M., \& Gómez-Olivé, F. X. (2017). Quality of integrated chronic disease care in rural South Africa: user and provider perspectives. Health policy and planning, 32(2), 257-266

Chang H. (2015). Evaluation Framework for Telemedicine Using the Logical Framework Approach and a Fishbone Diagram. Healthcare informatics research, 21(4), 230-238.

https://doi.org/10.4258/hir.2015.21.4.230 
Darcourt, J. G., Aparicio, K., Dorsey, P. M., Ensor, J. E., Zsigmond, E. M., Wong, S. T., ... \& Chang, J. C. (2021). Analysis of the implementation of telehealth visits for care of patients with cancer in Houston during the COVID-19 pandemic. JCO oncology practice, 17(1), e36-e43.

DeNysschen, C. A., Burton, H., Ademuyiwa, F., Levine, E., Tetewsky, S., \& O'connor, T. (2014).

Exercise intervention in breast cancer patients with aromatase inhibitor-associated arthralgia: a pilot study. European journal of cancer care, 23(4), 493-501.

Dietz, J. R., Moran, M. S., Isakoff, S. J., Kurtzman, S. H., Willey, S. C., Burstein, H. J., ... \& Stevens, R. E. (2020). Recommendations for prioritization, treatment, and triage of breast cancer patients during the COVID-19 pandemic. the COVID-19 pandemic breast cancer consortium. Breast cancer research and treatment, 181(3), 487.

Doyle, N. (2008). Cancer survivorship: evolutionary concept analysis. Journal of Advanced Nursing, 62(4), 499-509.

Dulko, D., Pace, C. M., Dittus, K. L., Sprague, B. L., Pollack, L. A., Hawkins, N. A., \& Geller, B. M. (2013). Barriers and facilitators to implementing cancer survivorship care plans. Oncology nursing forum, 40(6), 575-580. https://doi.org/10.1188/13.ONF.575-580

Elkaddoum, R., Haddad, F. G., Eid, R., \& Kourie, H. R. (2020). Telemedicine for cancer patients during COVID-19 pandemic: between threats and opportunities.

Eysenbach, Gunther, Dawn Dowding, Jane M Carrington, Pauline Johansson, Geneviève Rouleau, Marie-Pierre Gagnon, José Côté, Julie Payne-Gagnon, Emilie Hudson, and Carl-Ardy Dubois. "Impact of Information and Communication Technologies on Nursing Care: Results of an Overview of Systematic Reviews." Journal of Medical Internet Research 19.4 (2017): E122. Web. 
Hamer, J., \& Warner, E. (2017). Lifestyle modifications for patients with breast cancer to improve prognosis and optimize overall health. Cmaj, 189(7), E268-E274.

Hilgart, J. S., Hayward, J. A., Coles, B., \& Iredale, R. (2012). Telegenetics: a systematic review of telemedicine in genetics services. Genetics in Medicine, 14(9), 765-776.

Huguley Jr, C. M., \& Brown, R. L. (1981). The value of breast self-examination. Cancer, 47(5), 989995.

Iacoviello, L., Bonaccio, M., de Gaetano, G., \& Donati, M. B. (2020, February). Epidemiology of breast cancer, a paradigm of the" common soil" hypothesis. In Seminars in Cancer Biology. Academic Press.

National Research Council. (2005). From cancer patient to cancer survivor: lost in transition.

Jacobs, L. A., \& Shulman, L. N. (2017). Follow-up care of cancer survivors: challenges and solutions. The Lancet Oncology, 18(1), e19-e29.

Klevos, G. A., Ezuddin, N. S., Vinyard, A., Ghaddar, T., Gort, T., Almuna, A., ... \& Welsh, C. F. (2017). A breast cancer review: through the eyes of the doctor, nurse, and patient. Journal of Radiology Nursing, 36(3), 158-165.

Koonin, L. M., Hoots, B., Tsang, C. A., Leroy, Z., Farris, K., Jolly, B., ... \& Harris, A. M. (2020). Trends in the use of telehealth during the emergence of the COVID-19 pandemic-United States, January-March 2020. Morbidity and Mortality Weekly Report, 69(43), 1595.

Kruse, C. S., Krowski, N., Rodriguez, B., Tran, L., Vela, J., \& Brooks, M. (2017). Telehealth and patient satisfaction: a systematic review and narrative analysis. BMJ open, 7(8), e016242. 
Kumarasamy, H., Veerakumar, A. M., Subhathra, S., Suga, Y., \& Murugaraj, R. (2017). Determinants of awareness and practice of breast self-examination among rural women in Trichy, Tamil Nadu. Journal of mid-life health, 8(2), 84 .

Larson, J. L., Rosen, A. B., \& Wison, F. A. (2019). The effect of telehealth interventions on quality of life of cancer survivors: a systematic review and meta-analysis. Health Informatics Journal, 119. http://dx.doi.org/10.1177/1460458219863604

Leite, H., Gruber, T., \& Hodgkinson, I. R. (2020, April 8, 2020). Flattening the infection curve understanding the role of telehealth in managing COVID-19. Emeral Insight. Retrieved from https://www.emerald.com/insight/1751-1879.htm

Manocchia, A. (2020). Telehealth: Enhancing Care through Technology. Rhode Island Medical Journal, 103(1), 18-20.

Mayer, E. L., Gropper, A. B., Neville, B. A., Partridge, A. H., Cameron, D. B., Winer, E. P., \& Earle, C. C. (2012). Breast cancer survivors' perceptions of survivorship care options. Journal of Clinical Oncology, 30(2), 158.

National Research Council. (2005). From cancer patient to cancer survivor: lost in transition. National Academies Press.

Neeman, E., Kolevska, T., Reed, M., Sundaresan, T., Arora, A., Li, Y., ... \& Liu, R. (2020). Abstract S06-03: Cancer care telehealth utilization rates and provider attitudes in the wake of the novel coronavirus pandemic: The Kaiser Permanente Northern California experience.

Orlando, J. F., Beard, M., \& Kumar, S. (2019). Systematic review of patient and caregivers' satisfaction with telehealth videoconferencing as a mode of service delivery in managing patients' health. PloS one, 14(8), e0221848. 
Paterson, C., Bacon, R., Dwyer, R., Morrison, K. S., Toohey, K., O'Dea, A., ... \& Hayes, S. (2020, October). The role of telehealth during the COVID-19 pandemic across the interdisciplinary cancer team: Implications for practice. In Seminars in oncology nursing (p. 151090). WB Saunders.

Post, K. E., Moy, B., Furlani, C., Strand, E., Flanagan, J., \& Peppercorn, J. M. (2017). Survivorship Model of Care: Development and implementation of a nurse practitioner-led intervention for patients with breast cancer. Clinical journal of oncology nursing, 21(4).

Powell, R. E., Henstenburg, J. M., Cooper, G., Hollander, J. E., \& Rising, K. L. (2017). Patient perceptions of telehealth primary care video visits. The Annals of Family Medicine, 15(3), 225229.

Rand Health Care. (2018). Visit-specific satisfaction instrument (VSQ-9). Retrieved from: https://www.rand.org/health/surveys_tools/vsq-9.html

Rising, K. L., Ward, M. M., Goldwater, J. C., Bhagianadh, D., \& Hollander, J. E. (2018). Framework to advance oncology-related telehealth. JCO Clinical Cancer Informatics, 2, 1-11.

Rockwell, K. L., \& Gilroy, A. S. (2020). Incorporating telemedicine as part of COVID-19 outbreak response systems. Am J Manag Care, 26(4), 147-148.

Rosales, A. R., Byrne, D., Burnham, C., Watts, L., Clifford, K., Zuckerman, D. S., \& Beck, T. (2014). Comprehensive survivorship care with cost and revenue analysis. Journal of Oncology Practice, 10(2), e81-e85.

Runowicz, C. D., Leach, C. R., Henry, N. L., Henry, K. S., Mackey, H. T., Cowens-Alvarado, R. L., ... \& Hurria, A. (2016). American cancer society/American society of clinical oncology breast cancer survivorship care guideline. CA: a cancer journal for clinicians, 66(1), 43-73. 
Segelov, E., Underhill, C., Prenen, H., Karapetis, C., Jackson, C., Nott, L., ... \& Steer, C. Practical considerations for the management of cancer patients during the COVID19 pandemic.

Shapiro, C. L. (2018). Cancer survivorship. New England Journal of Medicine, 379(25), 2438-2450.

Spears, J.A., Craft, M., \& White, S. (2017). Outcomes of cancer survivorship care provided by advanced practice RNs compared to other models of care: A systematic review [Online exclusive]. https://doi.org/10.1188/17.ONF.E34-E41

Sprague, S. L., \& Holschuh, C. (2019, December). Telemedicine versus clinic visit. Clinical Journal of Oncology Nursing, 23, 639-646. Retrieved from 10.1188/19

Washington, P. K., Tews, H., Nguyen, D. T., Wade, L., Phan, T., Reddy, M. N., ... \& Shim, V. (2017). Use of telemedicine in the delivery of survivorship care plans among breast cancer survivors: Lessons learned at Kaiser Permanente East Bay.

Watson, P. W., \& McKinstry, B. (2009). A systematic review of interventions to improve recall of medical advice in healthcare consultations. Journal of the Royal Society of Medicine, 102(6).

World Health Organization. (2021). COVID-19 weekly epidemiological update, 13 March $2021.235-243$. 
APPENDICES 


\section{APPENDIX A}

\section{Survivorship clinic survey}

Due to COVID-19 we have switched to Telehealth (telephone or video) visits. We are asking you to fill out this survey because you had an initial meeting with your nurse practitioner to review your survivorship care plan and we would like to evaluate your satisfaction with your telehealth visit. We will only analyze summary data for all participants and we will not share your personal information with anyone. This project will also be used to meet academic degree requirements for the student investigator. All identifying information such as your name and medical record number will not be disclosed. Participation is voluntary. Choosing not to participate will not affect your care in any way. Regardless of your decision, we thank you for your time and consideration.

\section{How long ago was your survivorship visit?}

within the last 4 weeks

within the last 3 months

over 3 months ago

2.Please rate your level of satisfaction:

Very

ExcellentgoodGoodFairPo

Convenience of the telehealth appointment

Time spent with the nurse practitioner you spoke with

Technical skills (thoroughness, carefulness, competence) of the nurse practitioner you spoke with

The personal manner (courtesy, respect, sensitivity, friendliness) of the person you spoke with

Overall satisfaction with the visit

3.Please describe the information you recall from your survivorship visit:

Discussed,

just the

Discussed,right level Discussed

I do not but not

of too much rememberenough informationinformatic

Did you discuss your cancer stage and type?

Did you discuss your follow up plan (future office visits, surveillance tests)? 
Did you discuss healthy life-style recommendations (diet, nutrition, exercise, vitamins, bone health)?

Were you offered resources (dietitian, social work, support groups, physical therapy)?

Did you review your treatments (surgery, chemotherapy, radiation, medications)?

Did you discuss possible long term side effects from your treatments (or existing side effects)?

Did you discuss the importance of self breast exams?

4.Are you comfortable doing self breast exams?

$\checkmark$ Yes

5.Please rank the information discussed in your survivorship visit from MOST useful to LEAST useful. Click on your choice and move the arrow up or down putting what you valued the MOST on top and the LEAST on bottom.

Cancer stage and type

Follow-up plan

Healthy life-style recommendations

Resources offered

Review of your treatments

Possible long term side-effects from your treatment

6.Please choose your answer for each statement:

Strongly

Strongly

agree

AgreeNeutralDisagreedisagree N/

I like getting information through kp.org email.

I am comfortable doing my survivorship visits through telehealth (telephone or video).

Email instead of telephone or video visits would have been just fine.

7.Do you prefer telephone visits?

C Yes

No

8.Please answer why you said yes or why you said no in question \#7

9.Do you prefer video visits?

Y Yes 
No

10.Please answer why you said yes or why you said no in question \#9

11.What is your age?

less than 40

41-55

56-65

66-75

greater than 75

Submit 


\section{APPENDIX B}

Visit Specific Satisfaction Instrument

Thinking about your visit with the physician/health care Professional you saw, how would you rate the following: Poor Fair Good Very Good Excellent

1. How long you waited to get an appointment

2. Convenience of the location of the office

3. Getting through to the office by phone

4. Length of time waiting at the office

5. Time spent with the physician/health care professional you saw

6. Explanation of what was done for you

7. Technical skills (thoroughness, carefulness, competence) of the physician/health care professional you saw

8. The personal manner (courtesy, respect, sensitivity, friendliness) of the person you saw

9. The visit overall 


\section{APPENDIX C}

Telephone Script

Hello, my name is I am a nurse practitioner in oncology at Kaiser. Am I speaking with ?

I am calling you because you had a survivorship clinic visit to review your survivorship care plan through telehealth and I am asking if you would like to participate in a survey over the phone. This survey will take approximately 10-15 minutes. Would you like to participate? (yes continue. No- stop. "Thank you for your time, have a nice day").

During COVID-19 we have switched to Telehealth (telephone or video) visits. We are asking you to participate in this survey because you had an initial meeting with your nurse practitioner to review your survivorship care plan and we would like to evaluate your satisfaction with your telehealth visit. Your personal information is only available to the project team, who already has access to your personal information to provide care. We will only analyze summary data for participants and we will not share your personal information with anyone. This project will also be used to meet academic degree requirements for the student investigators doctorate of nursing program. This survey is confidential. All identifying information such as your name and medical record number will not be disclosed. Participation is voluntary.

Move on to attachment \#2. Verbal read off questions on survivorship survey. Record all answers on Microsoft Survey. 


\section{APPENDIX D}

Kaiser Permanente Northern California Oncology Treatment Summary and Survivorship Care Plan Breast Cancer

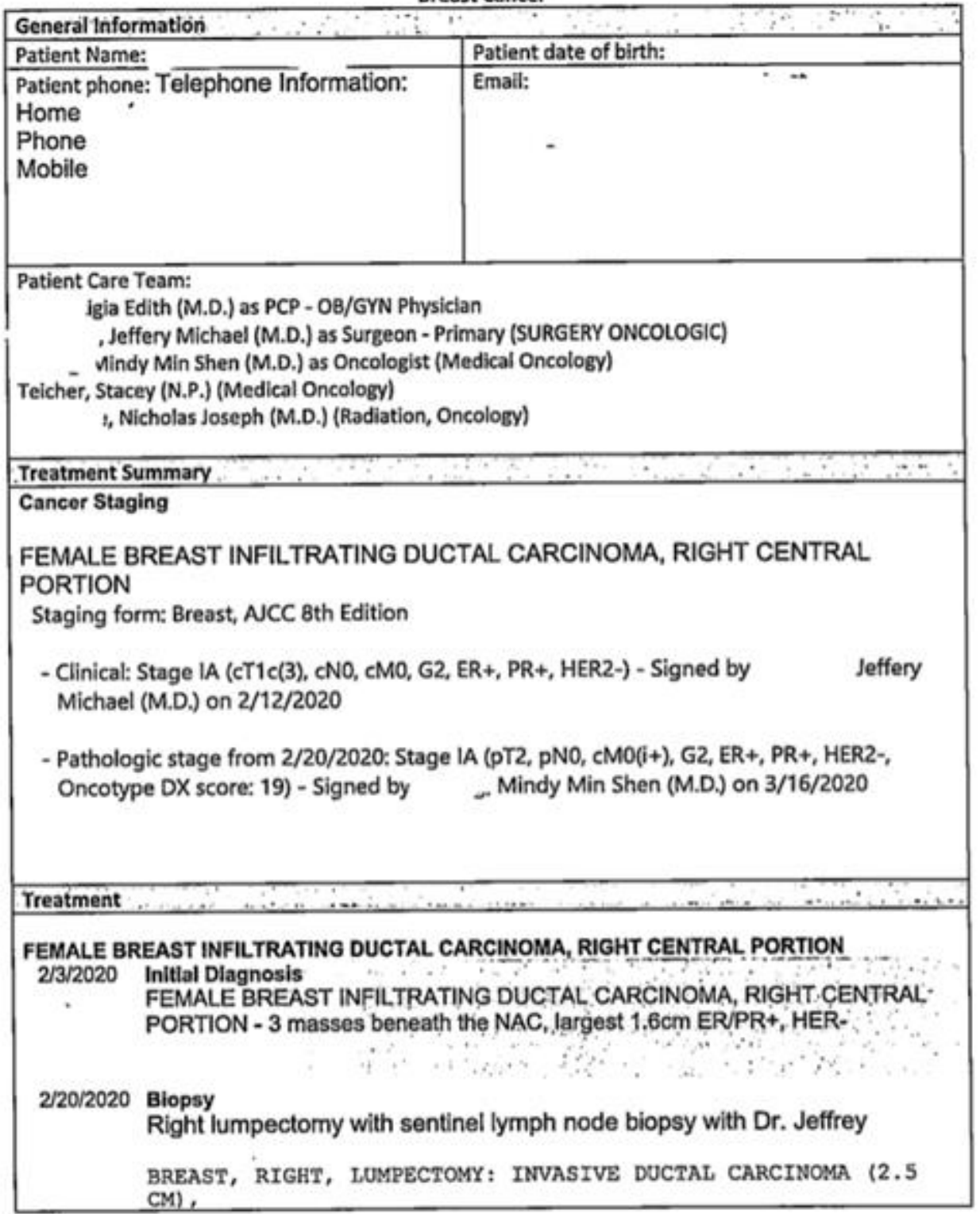


NOTTINGHAM GRADE 2.

0/8 LNs.

2/27/2020 Medical Oncology Treatment

-

Arimidex $1 \mathrm{mg}$ daily (anticipate 5 years)

6/4/2020 - Radiation

$6 / 24 / 2020$

\begin{tabular}{|c|c|c|c|c|c|c|c|c|c|}
\hline $\begin{array}{l}\text { Treatment } \\
\text { Site }\end{array}$ & Ref. ID & Energy & \begin{tabular}{|l|} 
Dose/Fx \\
(cGy)
\end{tabular} & HFx & $\begin{array}{l}\text { Dose } \\
\text { Correction } \\
\text { (cGy) }\end{array}$ & \begin{tabular}{|l} 
Total \\
Dose \\
(cGy)
\end{tabular} & \begin{tabular}{|l|} 
Start \\
Date \\
\end{tabular} & End Date & $\begin{array}{l}\text { Elapse } \\
\text { d Days }\end{array}$ \\
\hline Breast R & $\begin{array}{l}\text { Breast } \\
\mathbb{R}\end{array}$ & $15 \mathrm{X} / 6 \mathrm{x}$ & 267 & \begin{tabular}{|l|}
15 \\
115 \\
\end{tabular} & 0 & 4,005 & $6 / 4 / 2020$ & $6 / 24 / 2020$ & 20 \\
\hline Total: & & & & & & 4,005 & $6 / 4 / 2020$ & $6 / 24 / 2020$ & 20 \\
\hline
\end{tabular}

$6 / 24 / 2020$ End of Treatment

Familial Cancer Risk Assessment

Genetic counseling: no

Genetic testing results: none

Follow-up Care Plan

The following are general guidelines. Your oncologist may adjust this plan depending on factors such as your cancer risk and other health problems.

You will have regular follow-ups which should include a breast exam every 6 months while on Arimidex, then yearly.

Symptoms of Recurrence: Signs and symptoms of recurrent breast cancer varies. Please maintain routine follow-up visits. Report progressively worsening bone pain, discomfort in your abdomen, shortness of breath, breast lumps or new signs and symptoms to your doctor.

Medication: It is very important that you take Arimidex (anastrazole) as prescribed. Do not stop your anticancer medication without talking to your doctors. Skipping these anticancer medications can increase chance of cancer recurrence. Please talk to your providers if you have any concerns about your medications or symptoms.

Mammograms: Yeariy unless you had a mastectomy. 
Blood tests or $\mathrm{x}$-rays: Routine blood tests and $\mathrm{x}$-rays are not recommended because studies have shown they are not helpful.

Bone density: Periodically as needed and directed by your providers.

Please continue to see your primary care provider for all routine health care, including cancer screening tests. Report unusual vaginal bleeding to your oncologist and gynecologist.

Healthy Liféstyle.Choices

-Maintaining a healthy lifestyle is important for reducing the chance of cancer recurrence.

- Eat a healthy diet with an emphasis on plant sources, such as vegetables, fruits and whole grains.

- Adopt a physically active lifestyle. Exercise regularly. Some may benefit from weight bearing exercise

- Maintain a healthy body weight.

- Do not smoke or use tobacco products. If you do, we can help you quit. Please call

- (866) 251-4514 to set up a phone appointment.

- Limit your alcohol intake.

- Protect your skin from the sun by wearing hats or other clothing and using sunscreen.

- Supplement calcium and Vitamin D to maintain bone health if you are postmenopausal.

- No other vitamins or supplements are needed and some might be harmful.

Possible Effects of Cancer Treatment that you may experience after treatments

- Surgery: Lymphedema (limb swelling), scarring and pain on the surgical site

- Hormone therapy: Onset of early menopause, decreased appetite, joint pain, loss of libido (sexual desire)

- Radiation: Fatigue, discomfort, changes in skin coloration or texture, shrinkage or swelling or increased firmness of the treated area

- Mood: A diagnosis of cancer can affect your mood. Feelings of anxiety or depression are common. It can also affect your relationships, as well as lead to challenges with work and finances. Your primary provider who works with a team that includes a behavioral medicine specialist can help. 


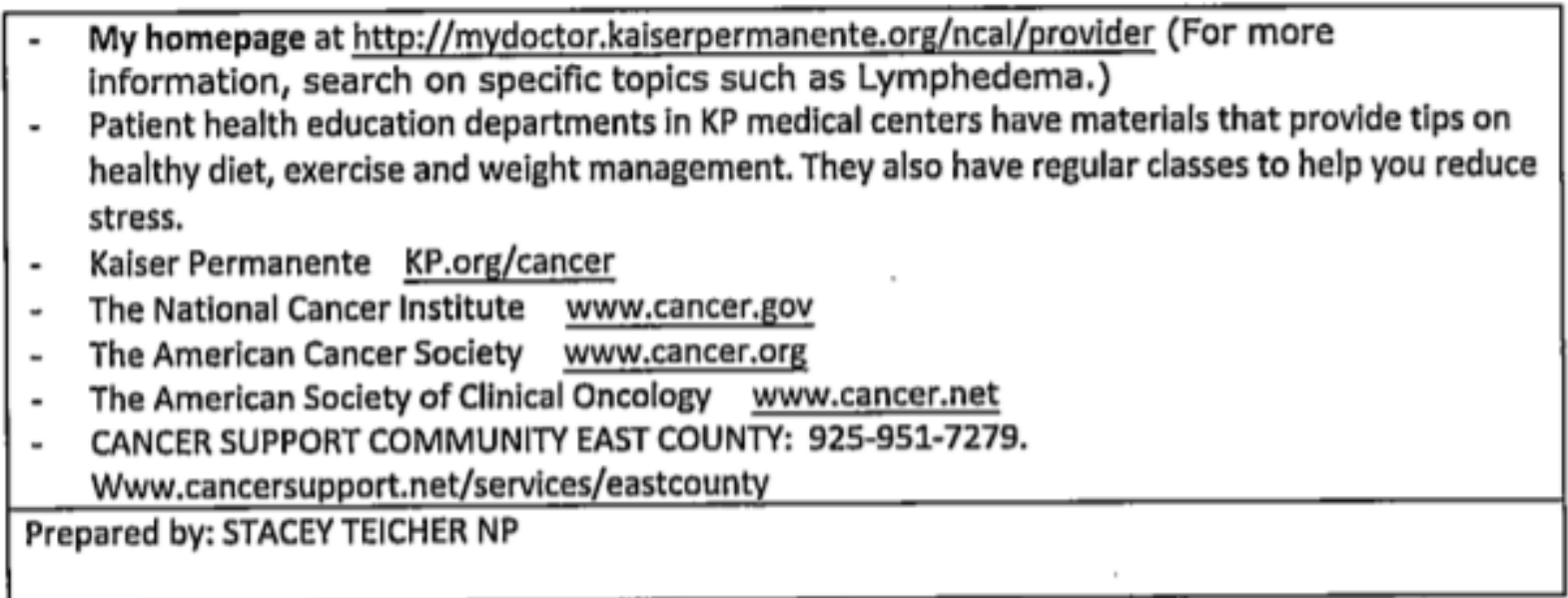

This is a brief summary of major aspects of your cancer treatment and plan for follow-up.

Please keep this with your health records. 


\section{APPENDIX E}

Date: June 29, 2020

Subject: RDO KPNC 20 - 100

Title: The effects of Telehealth on patient satisfaction and information recall for breast cancer survivors during COVID-19

Dear Dr. Liu:

The Research Determination Committee for the Kaiser Permanente Northern California region has reviewed the documents submitted for the above referenced project. The project does not meet the regulatory definition of research involving human subjects as noted here:

Not Research

The activity does not meet the regulatory definition of research at 45 CFR 46.102(d): Research means a systematic investigation, including research development, testing and evaluation, designed to develop or contribute to generalizable knowledge.

This determination is based on the information provided. If the scope or nature of the project changes in a manner that could impact this review, please resubmit for a new determination. The word "research" should not appear in any posters or publications resulting from this project. Further, if publications, presentations or posters are generated from this project the following wording must be used to reference to the project research determination outcome: "The Research Determination Committee for the Kaiser Permanente Northern California region has determined the project does not meet the regulatory definition of research involving human subjects per 45 CFR 46.102(d)" 
You are expected, however, to implement your study or project in a manner congruent with accepted professional standards and ethical guidelines as described in the Belmont Report (http://www.hhs.gov/ohrp/humansubjects/guidance/belmont.html). Additionally, you are responsible for keeping a copy of this determination letter in your project files as it may be necessary to demonstrate that your project was properly reviewed. Provide this approval letter to the Physician in Charge (PIC), your Area Manager, and Chief of Service, to determine whether additional approvals are needed.

Sincerely,

The Research Determination Committee KPNC-RDO@kp.org 
Appendix F

Please answer why you said yes or why you said no in question 7

Depending on why I'm seeing a nurse practitioner a phone visit would be fine. If it is a more serious or complicated issue then a video visit would be better.

Just prefer telephone

Convenience

To be able to communicate better. Sometimes message doesn't get across properly in emails.

I like to look people in the eyes when I speak to them. I prefer to ready farcical and body language.

Both phone and video visits are fine, I do not have a preference

I prefer in office visits, secondly are video visits.

I prefer either video visit or a visit in person

convenience

Face to face just seems so much more personal.

Because I can ask what I want to know and get an answer immediately 
Too impersonal

Prefer to see the person's face.

I like to see who I am speaking with

I like to see who I am talking to

Because I don't prefer them but I'm ok with them if I don't need to be seen.

I thought the initial visit by phone was fine. But for the rest of the visits I prefer in person.

Telephone better than emails because of inherent possibility for miscommunication.

Tone of voice, quickness of a response, among other cues, help me understand what is being communicated and whether or not, I should ask a follow up question. The same would be true on Kaiser's end of the call or email.

Prefer in person or video

Prefers VAV rather than TAV - Feels comfortable

Just because of COVID. After this is over, I don't mind going in. Although this is very convenient.

I prefer telephone visits over emails because it is a more personal form of communication.

Speaking to someone helps you understand what you might not otherwise.

I prefer to see the person I am speaking with because I then I am a complete person and not just a voice. 
During these times, telephone visits are great.

Video is my favorite.

Convenient, personal time valuable to me.

I prefer video visits over telephone visits because I make a more personal connection with my health care providers.

If the issue is minor, the telephone is fine. If it's a first visit or I have a pressing concern, phone visits don't work. Rather than an either or approach, I prefer what I call a "both and" approach.

Felt like I was connecting to a great person lots better then a phone call, like seeing the professional person.

I think is is important to be able to speak to your provider face to face.

\section{Impersonal}

This was such a relaxed conversation about such an important issue as breast cancer and its effects of the treatments.

Prefer video visit.

Convenience, safety during pandemic

personal and if something came up in the conversation, it could easily be addressed. Felt confident with the NP.

It's nice to see the care provider and for her to see me. 
Prefer in-person, but telephone is better than nothing

convient

Does not have a computer

Convenience

It is easier to communicate with my doctor and if $I$ have a question they can answer it on the spot.

I prefer to see the person I am dealing with face-to-face and think in-person is more beneficial at least the first time. Afterward telephone visits are $\mathrm{OK}$, however, person cannot check my breasts if needed and I prefer a breast check too.

I like to see my doctor in person I feel I get more out of my visit and its more personable

I answered no fpr this specific type of appointment. This is not a routine appt.

Because of my work schedule, it makes it more difficult to find time to have video chat. It is easier for me to leave work for a doctors appointment. More privacy in the doctors office than at work.

It is impersonal and a breast exam was not given by a medical professional. Breast Cancer survivor should always be given an exam.

I prefer talking in person with someone lnstead of reading emails. That way if a question pops up I can get an immediate answer.

I want a physical exam every time I go in person and telephone is lacking that!

I like in-person office visits.

My video chat drops in mid-appointment, so telephone is less hassle. 
I need a translator

For now I do. I prefer to see the doctor or nurse Practitioner in person. Hope we can do that soon.

I like the visual contact with my heath provider.

not technically inclined with video appointments

Personal touch is beter

I prefer Video visits to have a face to face interaction

Please answer why you said yes or why you said no in question 9

If you are not feeling well to go to the office visit, video visits are great.

Just video or telephone

Initially I prefer in-person appts, but for follow-up care either phone or video is great. Video feels more personal just because you can see who you're talking to.

I can see my doctor and am able to connect with them without having to come to the clinic and still be able to ask questions and voice my concerns.

More interactive then a phone call. Easier to engage and stay attentive 
Both phone and video visits are fine, I do not have a preference

Video visits are fine for some instances, but in person visits are preferable.

I can show the medical provider anything that I may not be able to describe properly over the phone

personable

Much more convenient. No travel and no exposure to the sick people at the clinic.

I don't feel they're are necessary

In person visits only

I can see the person's facial expressions.

Same answer as 8

I like to see who I am talking to

I prefer them over telephone visits but I'm ok with either.

Feel uncomfortable on video.

See above

Video appointments are okay when they are not physical issues that need to seen. 
communication videos easier than going in unless its a physical exam.

Same as above. COVID otherwise I don't mind coming in for an appointment.

I like being able to see who I am speaking with because it feels more personal.

Again I like to hear it from the Doctor or NP

I would rather have a face to face visit with my providers. My second choice is a video visit followed by a telephone visit

Not good with video visits .

I appreciate seeing the dr. or nurse. I feel I actually hear better when I see the person

Have not had a video visit that I recall.

See \#8.

In a video visit there is the opportunity to make a personal connection much more than on the phone.

Like seeing who I am talking too.

Again I think it is important to be able to see your provider.

Almost like actual face to face visit. Good for sharing information. Saves time and gas from actual clinic visit when no physical examination required.

Same as number 7 the wonder nurse practitioner was very reassuring and pleasant.

More personal to actually see medical personnel! 
So much better than office visits

Convenience, safety

Video visits are distracting - unable to take notes.

Same as 9.

I don't think I can figure out how to do this

gets complicated

Does not have a computer

Convenience

It is easier for me and my doctor to communicate and ask questions if I have any.

Sometimes the quality of video or audio is not great. And I have experiences issues connecting, both myself and the other person.

So I can at least see my doctor.

I prefer the video over telephone

If I am not at home, it is difficult to find a private area at work for me to chat to the level I feel comfortable.

No breast exam given by medical professional.

Video visits are closer to an actual office visit. It's best when you can actually see the person with whom you are talking to. 
There was NO physical exam which should be mandatory for breast cancer survivors!

two impersonal/

See answer for \#8

Translator too

I have never had a video visit, but, I open to it short term.

I like the visual contact with my heath provider.

Its unnerving because I do not know what I am doing.

Have to borrow a laptop

I feel comfortable and at ease with face to face interaction 